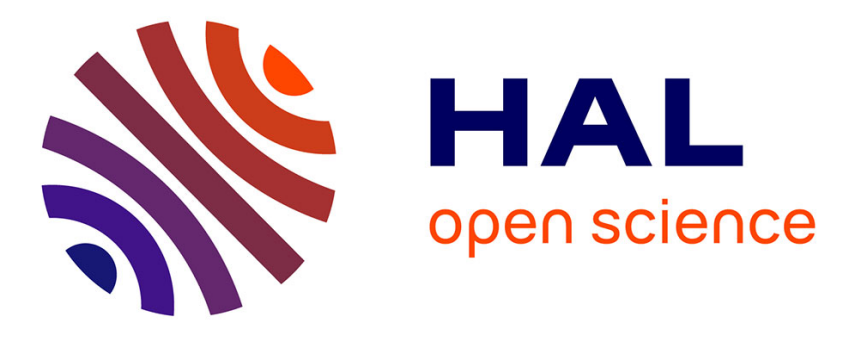

\title{
Reduced order models for resonant viscosity and mass density sensors
}

\author{
Martin Heinisch, T. Voglhuber-Brunnmaier, Erwin K. Reichel, Isabelle \\ Dufour, Bernhard Jakoby
}

\section{- To cite this version:}

Martin Heinisch, T. Voglhuber-Brunnmaier, Erwin K. Reichel, Isabelle Dufour, Bernhard Jakoby. Reduced order models for resonant viscosity and mass density sensors. Sensors and Actuators A: Physical , 2014, 214, pp.245-251. 10.1016/j.sna.2014.09.006 . hal-01062267

\section{HAL Id: hal-01062267 https://hal.science/hal-01062267}

Submitted on 13 Nov 2014

HAL is a multi-disciplinary open access archive for the deposit and dissemination of scientific research documents, whether they are published or not. The documents may come from teaching and research institutions in France or abroad, or from public or private research centers.
L'archive ouverte pluridisciplinaire HAL, est destinée au dépôt et à la diffusion de documents scientifiques de niveau recherche, publiés ou non, émanant des établissements d'enseignement et de recherche français ou étrangers, des laboratoires publics ou privés. 


\title{
Reduced Order Models for Resonant Viscosity and Mass Density Sensors
}

\author{
M. Heinisch ${ }^{\mathrm{a}}$, T. Voglhuber-Brunnmaier ${ }^{\mathrm{a}, \mathrm{b}}$, E.K. Reichel ${ }^{\mathrm{a}}$, I. Dufour ${ }^{\mathrm{c}}$, B. Jakoby ${ }^{\mathrm{a}}$ \\ ${ }^{a}$ Institute for Microelectronics and Microsensors, Johannes Kepler University, Linz, Austria \\ ${ }^{b}$ Center for Integrated Sensors Systems, Danube University, Krems, Austria \\ ${ }^{c}$ Université de Bordeaux, Laboratoire de l'Intégration du Matériau au Système, France
}

\begin{abstract}
A generalized reduced order model for resonant viscosity and mass density sensors is presented and experimentally verified. The reduced expressions for the resonance frequency and quality factor, respectively, are mathematically valid for in-plane oscillating plates, oscillating spheres and laterally oscillating cylinders. However, as shown for measurements obtained with a tuning fork resonator with rectangular cross-section, the model can also be applied for resonating structures, for which closed form solutions of the fluid structure interaction are not available. Benefits of the presented model are amongst others first, its simplicity, which requires no more than three calibration measurements for parameter identification and second, its general applicability for resonant mass density and viscosity sensors which furthermore facilitates the comparison of different resonant mass density and viscosity sensors in terms of sensitivity and measurement noise propagation.
\end{abstract}

Keywords: Lumped element model, resonant, sensor, oscillating, plate, sphere, cylinder, tuning fork

\section{Introduction}

During the last two decades, there has been a remarkable interest in resonant viscosity and mass density sensors. As a matter of fact, this interest is still increasing which is substantiated by the large amount of publications associated with this field. Some of the incentives for resonant viscosity and mass density sensors are, amongst others, their reduced size compared to conventional instruments (down to the micrometer range), their relatively straightforward integrability in a process line or potentially low manufacturing costs [1]. Furthermore, from a rheological point of view, these devices allow measurements in a frequency range higher than conventional rheometers (about $100 \mathrm{~Hz}$ ) up to the $\mathrm{MHz}$ range, which becomes especially interesting when investigating linear viscoelastic liquids for which measurements in the mentioned frequency range are scarcly available yet. Finally, for many resonant principles, miniaturized devices can be fabricated, and thus these sensors allow characterizing liquid samples for which only very few amounts (lower than $1 \mathrm{~nL}$ ) are available [2] .

The variety of reported designs and fabrication technologies is extremly diverse. Common principles include thickness shear mode oscillating quartz crystals, [3], [4], quartz tuning forks [5] and singly [6] or doubly clamped beams [7] in silicon technology. Similar devices were also fabricated using wet-etched copper coated polymer sheets [8] or longitudinally vibrating PZT screen-printed cantilevers [9]. For frequencies between $100 \mathrm{~Hz}$ and $100 \mathrm{kHz}$ and similar to doubly clamped beams, wire based sensors [10], [11] were reported. Other principles also operating in the aforementioned frequency range include U-shaped cantilevers [12], [13], membrane based devices [14], [15] and in-plane oscillating platelets [16], [17], [18].

Concerning excitation and readout, the previously reported concepts cover most established principles used in MEMS technology. Recording the frequency responses of piezoelectric or piezoresistive devices [19], [9], [6] is a very common technique. In [20] a capacitive readout principle was reported. Electromagnetic principles allow high driving forces and large deflections [21] as well as measuring the frequency response by recording a motion induced voltage [14] at the same time. In [22], [23], [24] thermally actuated devices are discussed which allow an in-plane oscillation of the particular device. Optical readout using e.g. a laser vibrometer [25] is a further very common technique, though hardly suitable for integration in a sensor device used in the industrial field and only applicable for transparent liquids. Due to their high accuracy in deflection measurements, laser vibrometers are often used for the readout of miniaturized cantilever based setups e.g. in biosensing applications [26] in a laboratory environment. In [7] an optical readout using a DVD-pickup was implemented and in [27] a setup with a closed fluid cell using optical readout is presented. For all types of liquid sensors, closed setups are a basic requirement when aiming at accurate measurement results. Open setups usually involve evaporation of the liquid, which in turn is one of the sources for non-stable measuring conditions as the liquid's temperature varies due to evaporative cooling. This variation of temperature during the measurement involves mostly unknown changes of the resonator's mechanical parameters but also changes of the liquid's mass density and viscosity.

The principles which were discussed in the previous paragraphs have in common, that the devices' frequency responses, including a characteristic resonant mode, are recorded from which the sample liquid's mass density $\rho$ and viscosity $\eta$ are deduced. In many cases, resonance frequency $f_{\mathrm{r}}$ and quality factor 
$Q$ are first evaluated, which are then related to the liquid's viscosity and mass density by an appropriate model, see Fig.1. Depending on the particular resonator design, closed form models considering structural and fluid mechanics may become relatively complex demanding high modelling effort and computational power. Furthermore, the parameters in these models have to be identified with an appropriate parameter fit or the model might have to be completed by calibration functions which require additional calibration measurements, see e.g. [12]. In addition to this high modeling and calibration effort, there is a lack of a generalized model for resonant mass density and viscosity sensors which can be used as figure of merit and allowing for comparison of different sensor principles.

In this paper, a generalized reduced order model is presented, relating resonance frequency $f_{\mathrm{r}}$ and quality factor $Q$ to mass density $\rho$ and viscosity $\eta$. The equations for the latter have the form

$$
\begin{aligned}
& f_{\mathrm{r}} \approx \frac{1 /(2 \pi)}{\sqrt{m_{0 \mathrm{k}}+m_{\rho \mathrm{k}} \rho+m_{\eta \rho \mathrm{k}}^{*} \sqrt{\eta \rho}}} \quad \text { and } \\
& Q \approx \frac{\sqrt{m_{0 \mathrm{k}}+m_{\rho \mathrm{k}} \rho+m_{\eta \rho \mathrm{k}}^{*} \sqrt{\eta \rho}}}{c_{0 \mathrm{k}}+c_{\eta \mathrm{k}} \eta+c_{\eta \rho \mathrm{k}}^{*} \sqrt{\eta \rho}}
\end{aligned}
$$

which will be derived in the following. $\left(m_{0 \mathrm{k}}, m_{\rho \mathrm{k}}, m_{\eta \rho \mathrm{k}}^{*}, c_{0 \mathrm{k}}\right.$, $c_{\eta \mathrm{k}}, c_{\eta \rho \mathrm{k}}^{*}$ are coefficients which can be determined with a parameter fit.) These generic equations can be derived for resonators where an infinitely thin, in-plane oscillating plate, an oscillating sphere or a laterally oscillating cylinder interact with the fluid. However, as it will be shown for measurements obtained with sensors e.g. with rectangular cross-section, this reduced order model can also be applied for geometries other than the aforementioned, for which analytical expression are not available or hardly accessible.

Assuming linearity for the mechanical oscillation, and thus small deflection amplitudes of the resonator [28], the investigated resonant mode is represented by a mechanical, lumped element oscillator, assuming validity of eigenmode decomposition. In the presented model, the fluid structure interactions are described in a reduced generalized expression, considering the well known equations for an in-plane oscillating plate, an oscillating sphere and a laterally oscillating cylinder. In this generalized model, any material or geometrical related parameters as well as any other factors are not explicitly considered but contained in single factors. Thereby, only the dependencies to mass density and viscosity remain in the derived expressions for the resonance frequency and the quality or damping factor. This basically allows the applicability of the model for any convexly shaped resonator, regardless of the implemented designs, materials or fabrication technologies as well as actuation and readout mechanisms.

Besides the simplicity of the model and its general applicability, a further big advantage is that only measurements in three calibration liquids are necessary to identify the model's coefficients. From every measurement $f \mathrm{r}$ and $Q$ are evaluated which then allows evaluating the six coefficients in the

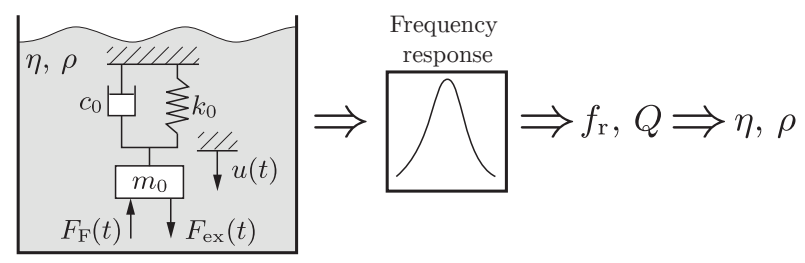

Figure 1: Mechanical, lumped elements oscillator, immersed into a liquid. $m_{0}$ : lumped mass, $c_{0}$ : damping coefficient, $k_{0}$ : spring constant, $u(t)$ : displacement, $F_{\text {ex }}(t)$ : excitation force, $F_{\mathrm{F}}(t)$ : fluid force, $f_{\mathrm{r}}$ : resonance frequency, $Q$ : Quality factor, $\eta$ : fluid's dynamic viscosity, $\rho$ : fluid's mass density

equations above. For resonant sensors whose identified model slightly deviates from the measurements, the model still can be used to approximately describe the sensor's characteristics and allows comparison with other principles which were identified with this generalized, reduced order model.

\section{Lumped element approach}

We consider a linear, mechanical oscillator, see Fig. 1, with lumped mass $m_{0}$, damping coefficient $c_{0}$ and spring constant $k_{0}$, being immersed in a liquid. The time dependent equation of motion with time variable $t$ for the displacement $u$ upon forced actuation with an excitation force $F_{\text {ex }}$ and accounting for the influence of the liquid on the oscillation with a fluid related force $F_{\mathrm{F}}$ is

$$
m_{0} \frac{\mathrm{d}^{2} u(t)}{\mathrm{d} t^{2}}+c_{0} \frac{\mathrm{d} u(t)}{\mathrm{d} t}+k_{0} u(t)=F_{\mathrm{ex}}(t)-F_{\mathrm{F}}(t) .
$$

A harmonic excitation force with angular frequency $\omega$ and time dependence $e^{\mathrm{j} \omega t}$ where $\mathrm{j}=\sqrt{-1}$ is furthermore assumed. Thus, the equation of motion using the steady state velocity of the oscillation in the frequency domain $\underline{v}(\omega)=j \omega \underline{u}(\omega)$ reads

$$
\left(c_{0}+j\left(\omega m_{0}-\frac{k_{0}}{\omega}\right)\right) \underline{v}(\omega)=\underline{F}_{\mathrm{ex}}(\omega)-\underline{F}_{\mathrm{F}}(\omega) .
$$

The force $\underline{F}_{\mathrm{F}}$ induced by the fluid can be represented by a complex valued function of $\omega$ in the frequency domain. As the interaction will have a linear character for small vibration amplitudes it will be proportional to the velocity such that we can write

$$
\underline{F}_{\mathrm{F}}(\omega)=\underline{Z}_{\mathrm{F}}(\omega) \underline{v}(\omega)
$$

where we used the symbol $\underline{Z}_{\mathrm{F}}$ for the proportionality factor as it resembles an acoustic impedance. $\underline{Z}_{\mathrm{F}}(\omega)$ can be split in a real and imaginary part, $Z_{\mathrm{F}, \mathfrak{R}}$ and $Z_{\mathrm{F}, \mathfrak{I}}$, respectively. Intuitively, the interaction with the fluid will lead to an added mass (due to fluid mass moved in unison with the vibration) and to a damping due to viscous losses and radiated acoustic waves. The latter effect will lead to a real part of $\underline{Z}_{\mathrm{F}}(\omega)$ while the mass entrainment corresponds to the imaginary part. This motivates to write the real part as

$$
Z_{\mathrm{F}, \mathfrak{R}}=c_{\mathrm{f}}
$$


and the imaginary part as

$$
Z_{\mathrm{F}, \mathfrak{I}}=\omega m_{\mathrm{f}},
$$

where $c_{\mathrm{f}}$ represents the additional damping and $m_{\mathrm{f}}$ represents the added mass. If additional lumped mass were actually added to the lumped mass $m_{0}$ of the mechanical oscillator, $m_{\mathrm{f}}$ would represent this added mass. However, as it represents the fluid interaction of a distributed resonator, the terms $m_{\mathrm{f}}$ and $c_{\mathrm{f}}$ will, in general, be frequency dependent. Thus the fluid forces in the frequency domain can be written as

$$
\underline{F}_{\mathrm{F}}(\omega)=\left(c_{\mathrm{f}}(\omega)+\mathrm{j} \omega m_{\mathrm{f}}(\omega)\right) \underline{v}(\omega) .
$$

Just as the parameters of the lumped mass model $\left(m_{0}, c_{0}\right.$, $\left.k_{0}\right)$, the frequency dependence of $m_{\mathrm{f}}(\omega)$ and $c_{\mathrm{f}}(\omega)$ can be moderate, though, such that, if $\omega$ is close to the resonance frequencies, the values can be approximated by their values at the resonance frequency.

Introducing the above relation Eq. 6 in Eq. 2 we obtain the following spectral transfer function

$$
\frac{\underline{v}}{\underline{F}_{\mathrm{ex}}}=\frac{1}{c_{0}+c_{\mathrm{f}}(\omega)+j\left(\omega\left(m_{0}+m_{\mathrm{f}}(\omega)\right)-\frac{k_{0}}{\omega}\right)} .
$$

This frequency response can be compared to the standardized response of a second order system

$$
\underline{H}_{2}(\omega)=\frac{A}{1+j Q\left(\frac{\omega}{\omega_{0}}-\frac{\omega_{0}}{\omega}\right)}
$$

where $\omega_{0}$ represents the resonance frequency and $Q$ the quality factor. Neglecting the frequency dependence of $\underline{Z}_{\mathrm{F}}$ near resonance, the resonance frequency and the Q-factor of our model system can be readily identified. The resonance frequency is defined as the frequency where the above transfer function Eq.7 becomes real-valued which yields an implicit equation for $\omega_{0}$ :

$$
\omega_{0}\left(m_{0}+m_{\mathrm{f}}(\omega)\right)=\frac{k_{0}}{\omega_{0}}
$$

This equation could, e.g. be solved iteratively using the approximation $m_{\mathrm{f}}(\omega) \approx m_{\mathrm{f}}\left(\omega_{00}\right)$ as a starting value for $m_{\mathrm{f}}(\omega)$ where $\omega_{00}=\sqrt{k_{0} / m_{0}}$ is the resonance eigenfrequency for the unloaded resonator. For weak frequency dependence of $m_{\mathrm{f}}(\omega)$ or small resonance frequency shifts, this first approximation may already be considered as sufficiently accurate (see also the discussion below for specific cases). With this simple approximation we find

$$
\omega_{0} \approx \omega_{01}=\sqrt{\frac{k_{0}}{m_{0}+m_{\mathrm{f}}\left(\omega_{00}\right)}}
$$

which represents the decrease of resonance frequency associated with the added mass as expected. $\omega_{01}$ is the approximated value for $\omega_{0}$ after the first iteration. Similarly, for $Q$ we find

$$
Q \approx Q_{1}=\frac{1}{\omega_{0}} \cdot \frac{k_{0}}{c_{0}+c_{\mathrm{f}}\left(\omega_{00}\right)} .
$$

The exact values for $\omega_{0}$ and $Q$ are

$$
\omega_{0}=\sqrt{\frac{k_{0}}{m_{0}+m_{\mathrm{f}}\left(\omega_{0}\right)}} \text { and } Q=\frac{1}{\omega_{0}} \cdot \frac{k_{0}}{c_{0}+c_{\mathrm{f}}\left(\omega_{0}\right)}
$$

yielding an implicit equation for $\omega_{0}$.

\section{Fluid forces}

\subsection{In-plane oscillating plate}

The shear stress $\underline{T}_{\mathrm{p}}$ resulting from the in-plane oscillation of an infinitely extended plate in a viscous liquid with dynamic viscosity $\eta$ and mass density $\rho$ at the liquid-solid interface in the frequency domain reads [3], [29]

$$
\underline{T}_{\mathrm{p}}=-(1+\mathrm{j}) \sqrt{\frac{\eta \rho \omega}{2}} \underline{v} .
$$

The fluid forces acting on the plate with effective surface $A_{\mathrm{p}}$ are $\underline{F}_{\mathrm{F}}=-\underline{T}_{\mathrm{p}} A_{\mathrm{p}}$. We call $A_{\mathrm{p}}$ effective surface, as this coefficient which might be determined by a data fit, considers not only the surface of wetted parts of a plane plate, but it might also consider geometrical imperfections such as slight surface roughnesses and plates with a finite thickness. Comparison of coefficients of the latter and Eq. 6 yields for the additional effective fluid mass and damping parameter in case of the in-plane oscillating plate

$$
m_{\mathrm{f}, \mathrm{p}}(\omega)=\sqrt{\frac{\eta \rho}{2 \omega}} A_{\mathrm{p}} \quad \text { and } \quad c_{\mathrm{f}, \mathrm{p}}(\omega)=\sqrt{\frac{\eta \rho \omega}{2}} A_{\mathrm{p}}(14)
$$

Introducing coefficients $m_{\eta \rho, p}=c_{\eta \rho, p}=A_{\mathrm{p}} / \sqrt{2}$ used in the expressions for the added mass and damping parameters in case of the plate, Eq. 14 gives

$$
m_{\mathrm{f}, \mathrm{p}}(\omega)=m_{\eta \rho, \mathrm{p}} \sqrt{\frac{\eta \rho}{\omega}} \quad \text { and } \quad c_{\mathrm{f}, \mathrm{p}}(\omega)=c_{\eta \rho, \mathrm{p}} \sqrt{\omega \eta \rho}(15)
$$

\subsection{Oscillating sphere}

The fluid forces acting on a sphere with radius $r_{\mathrm{s}}$, oscillating with velocity $v(t)$ is [30]

$$
\begin{aligned}
F_{\mathrm{F}, \mathrm{s}}= & 6 \pi \eta r_{\mathrm{s}}\left(1+\frac{r_{\mathrm{s}}}{\delta}\right) v(t) \\
& +3 \pi r_{\mathrm{s}}^{2} \sqrt{\frac{2 \eta \rho}{\omega}}\left(1+\frac{2 r_{\mathrm{s}}}{9 \delta}\right) \frac{\partial v(t)}{\partial t}
\end{aligned}
$$

where

$$
\delta=\sqrt{\frac{2 \eta}{\omega \rho}}
$$

is the so-called penetration depth.

Substituting Eq. 17 in Eq. 16, transformation to the frequency domain and comparison of coefficients with Eq. 6 yields 


$$
\begin{aligned}
& m_{\mathrm{f}, \mathrm{s}}(\omega)=3 \pi r_{\mathrm{s}}^{2}\left(\sqrt{\frac{2 \eta \rho}{\omega}}+\frac{2 r_{\mathrm{s}}}{9} \rho\right) \text { and } \\
& c_{\mathrm{f}, \mathrm{s}}(\omega)=6 \pi r_{\mathrm{s}}\left(\eta+r_{\mathrm{s}} \sqrt{\frac{\omega \eta \rho}{2}}\right) .
\end{aligned}
$$

Considering all coefficients and $r_{\mathrm{s}}$ in factors $m_{\mathrm{x}, \mathrm{s}}$ and $c_{\mathrm{x}, \mathrm{s}}$ used in the expressions for the fluid loading related additive mass and damping parameter in case of the sphere, Eq. 18 can be brought into the form

$$
\begin{aligned}
& m_{\mathrm{f}, \mathrm{s}}(\omega)=m_{\rho, \mathrm{s}} \rho+m_{\eta \rho, \mathrm{s}} \sqrt{\frac{\eta \rho}{\omega}} \text { and } \\
& c_{\mathrm{f}, \mathrm{s}}(\omega)=c_{\eta, \mathrm{s}} \eta+c_{\eta \rho, \mathrm{s}} \sqrt{\omega \eta \rho} .
\end{aligned}
$$

\subsection{Oscillating cylinder}

The fluid force per unit length $\underline{F}_{\mathrm{F}, \mathrm{c}}^{\prime}$ on an oscillating cylinder with radius $r_{\mathrm{c}}$, also given in [31], [32], [33], reads:

$$
\underline{F}_{\mathrm{F}, \mathrm{c}}^{\prime}(\omega)=-\pi \rho \omega^{2} r_{\mathrm{c}}^{2} \underline{\Gamma}_{\mathrm{cyl}} \underline{u}(\omega)
$$

with

$$
\underline{\Gamma}_{\mathrm{cyl}}=1+\frac{4 K_{1}(\sqrt{\mathrm{j} R e})}{\sqrt{\mathrm{j} R e} K_{0}(\sqrt{\mathrm{j} R e})}
$$

where

$$
\operatorname{Re}=\frac{\rho \omega r_{\mathrm{c}}^{2}}{\eta}
$$

is the Reynold's number, $K_{0}$ and $K_{1}$ are modified Bessel functions of second kind and $\underline{\Gamma}_{c y l}$ is the so-called hydrodynamic function.

To allow evaluating the real and imaginary part of Eq. 20, necessary for calculating added mass $m_{\mathrm{f}, \mathrm{c}}$ and damping parameter $c_{\mathrm{f}, \mathrm{c}}$ for the oscillating cylinder, a second order series expansion of $\underline{\Gamma}_{\mathrm{cyl}}$ at $j \operatorname{Re} \rightarrow \infty$ is performed, yielding

$$
\underline{\Gamma}_{\mathrm{cyl}} \approx \underline{\Gamma}_{\mathrm{cyl}, \mathrm{T}}=1+\frac{4}{\sqrt{2 R e}}-\mathrm{j}\left(\frac{4}{\sqrt{2 R e}}+\frac{2}{R e}\right)
$$

where the subscript $\mathrm{T}$ denotes the Taylor series developed expression. The relative deviations of real and imaginary parts $\delta \underline{\Gamma}_{\mathrm{cyl}, \mathfrak{R}}$ and $\delta \underline{\Gamma}_{\mathrm{cyl}, \mathfrak{J}}$ of the approximated hydrodynamic function Eq. 23 and the exact solution Eq. 21 is depicted in Fig. 2. This comparison shows that for Reynolds numbers larger than 10, the deviation of the Taylor series approximated solution is less than $1 \%$.

Substituting the approximated solution of the hydrodynamic function in Eq. 20 and comparison of coefficients with Eq. 6 yields

$$
\begin{aligned}
& m_{\mathrm{f}, \mathrm{c}}(\omega) \approx \pi r_{\mathrm{c}}^{2}\left(\rho+\frac{4}{\sqrt{2} r_{\mathrm{c}}} \sqrt{\frac{\eta \rho}{\omega}}\right) l_{\mathrm{c}} \text { and } \\
& c_{\mathrm{f}, \mathrm{c}}(\omega) \approx\left(2 \pi \eta+\frac{4 \pi}{\sqrt{2}} r_{\mathrm{c}} \sqrt{\omega \eta \rho}\right) l_{\mathrm{c}}
\end{aligned}
$$
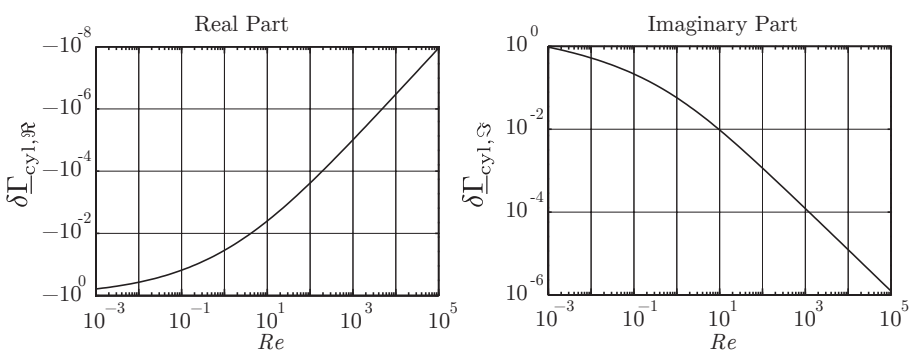

Figure 2: Relative deviations of the real and imaginary parts of the approximated hydrodynamic function Eq. 23 from the exact solution Eq. 21.

for the added mass and damping parameters in case of the oscillating cylinder, where $l_{\mathrm{c}}$ is the effective length of the cylinder. By reducing all coefficients and $r_{\mathrm{c}}$ in factors $m_{\mathrm{x}, \mathrm{c}}$ and $c_{\mathrm{x}, \mathrm{c}}$,

$$
\begin{aligned}
& m_{\mathrm{f}, \mathrm{c}}(\omega)=m_{\rho, \mathrm{c}} \rho+m_{\eta \rho, \mathrm{c}} \sqrt{\frac{\eta \rho}{\omega}} \text { and } \\
& c_{\mathrm{f}, \mathrm{c}}(\omega)=c_{\eta, \mathrm{c}} \eta+c_{\eta \rho, \mathrm{c}} \sqrt{\omega \eta \rho}
\end{aligned}
$$

is obtained for the oscillating cylinder which is the same form as Eq. 19 for the oscillating sphere.

\subsection{Generalized equations for added mass and damping}

Comparison of the Eqs. 15, 19 and 25 shows that the frequency dependent expressions for the fluid related added mass and damping can be given in a generalized form as follows

$$
\begin{aligned}
& m_{\mathrm{f}}(\omega)=m_{\rho} \rho+m_{\eta \rho} \sqrt{\frac{\eta \rho}{\omega}} \text { and } \\
& c_{\mathrm{f}}(\omega)=c_{\eta} \eta+c_{\eta \rho} \sqrt{\omega \eta \rho}
\end{aligned}
$$

where the factors for $m_{\mathrm{x}}$ and $c_{\mathrm{x}}$ are given in Tab. 1 for the case of an in-plane oscillating plate, an oscillating sphere and a laterally oscillating cylinder.

\begin{tabular}{|c||c|c|c|}
\hline & Plate & Sphere & Cylinder \\
\hline \hline$m_{\rho}$ & 0 & $\frac{2 \pi}{3} r_{\mathrm{s}}^{3}$ & $\pi r_{\mathrm{c}}^{2} l_{\mathrm{c}}$ \\
\hline$m_{\eta \rho}$ & $\frac{A_{\mathrm{p}}}{\sqrt{2}}$ & $\sqrt{2} 3 \pi r_{\mathrm{s}}^{2}$ & $\sqrt{2} 2 \pi r_{\mathrm{c}} l_{\mathrm{c}}$ \\
\hline \hline$c_{\eta}$ & 0 & $6 \pi r_{\mathrm{s}}$ & $2 \pi l_{\mathrm{c}}$ \\
\hline$c_{\eta \rho}$ & $\frac{A_{\mathrm{p}}}{\sqrt{2}}$ & $\sqrt{2} 3 \pi r_{\mathrm{s}}^{2}$ & $\sqrt{2} 2 \pi r_{\mathrm{c}} l_{\mathrm{c}}$ \\
\hline
\end{tabular}

Table 1: Coefficients

\section{Generalized equations for resonance frequency and damp- ing factor for a liquid loaded oscillator}

\subsection{Resonance frequency}

Considering the three solutions for the fluid-loading related mass parameter, a generalized expression for the angular resonance frequency

$$
\omega_{0}=\frac{1}{\sqrt{m_{0 \mathrm{k}}+m_{\rho \mathrm{k}} \rho+m_{\eta \rho \mathrm{k}} \sqrt{\frac{\eta \rho}{\omega_{0}}}}}
$$


is obtained by substituting the mass parameter from Eq. 26 in Eq. 12 and dividing the such obtained equation by $k_{0} . m_{x \mathrm{k}}$ are coefficients which can be determined by a parameter fit for measurement results for $f_{\mathrm{r}}=\omega_{0} /(2 \pi)$ obtained with at least three different liquids with known mass densities and viscosities. In case of one-dimensional plane shear waves, the factor $m_{\rho \mathrm{k}}$ is zero.

\subsection{Quality factor}

Similar to the generalized equation for the resonance frequency, the quality factor is calculated by substituting the damping coefficient from Eq. 26 in Eq. 12. It follows

$$
Q=\frac{1}{\omega_{0}} \cdot \frac{1}{c_{0 \mathrm{k}}+c_{\eta \mathrm{k}} \eta+c_{\eta \rho \mathrm{k}} \sqrt{\omega_{0} \eta \rho}},
$$

where $c_{x \mathrm{k}}$ are coefficients and $c_{\eta \mathrm{k}}$ is zero in case of one-dimensional shear waves.

\section{Simplified equation for the resonance frequency and damp- ing factor}

\subsection{Resonance frequency}

The implicit nature of Eq. 27 might be troublesome in some cases, e.g. when an estimation for $\omega_{0}$ for given $\eta$ and $\rho$ has to be calculated. For this purpose, the equation is simplified by neglecting the frequency dependence of the added mass $m_{\mathrm{f}}(\omega)$ in Eq. 26. Thus, it follows for the angular resonance frequency

$$
\omega_{0}=\frac{1}{\sqrt{m_{0 \mathrm{k}}+m_{\rho \mathrm{k}} \rho+m_{\eta \rho \mathrm{k}}^{*} \sqrt{\eta \rho}}}
$$

\subsection{Quality factor}

Similar to the simplification of $\omega_{0}$, the expression for $Q$ can be simplified by neglecting the frequency dependence of the damping parameter $c_{\mathrm{f}}(\omega)$ in Eq. 26. Using Eq. 29 for $\omega_{0}$ in Eq. 28 , it follows

$$
Q=\frac{\sqrt{m_{0 \mathrm{k}}+m_{\rho \mathrm{k}} \rho+m_{\mathrm{\eta} \rho \mathrm{k}}^{*} \sqrt{\eta \rho}}}{c_{0 \mathrm{k}}+c_{\eta \mathrm{k}} \eta+c_{\eta \rho \mathrm{k}}^{*} \sqrt{\eta \rho}} .
$$

As already mentioned above, the simplification of the equations for $\omega_{0}$ and $Q$ by neglecting the frequency dependence of the parameters $m_{\mathrm{f}}(\omega)$ and $c_{\mathrm{f}}(\omega)$ is valid for small investigated bandwidths and high quality factors. In a first theoretical investigation which will be published elsewhere it was found, that for an investigated bandwidth of $\left[\omega_{0}-\omega_{0} / Q \ldots \omega_{0}+\omega_{0} / Q\right]$ the relative changes of $m_{\mathrm{f}}(\omega)$ and $c_{\mathrm{f}}(\omega)$ are less than $1 \%$ for quality factors higher than 5 in case of $m_{\mathrm{f}}$ and 50 in case of $c_{\mathrm{f}}$, respectively. A comparison of the generalized model and simplified model for fitted parameters is given in Sec.6 and in Appendix B.

The procedure how to fit the model parameters in Eqs. 27 30 using a linear least squares fit, is explained in Appendix A.

\section{Model Verification}

For investigating its validity, the model has been applied to experimental results obtained with steel tuning forks and to data of recently published sensors. The deviations of measured and modeled results for each sensor are depicted in Fig. B.6 in Appendix B.

The intention of the experiments performed for this work was to experimentally examine the effect of mass density and viscosity to resonance frequency and quality factor separately. This was achieved by investigating the resonance characteristics of tuning forks in liquid mixture series with varying viscosities but constant mass densities and vice versa. This procedure is explained in detail in the following subsection.

\subsection{Experimental investigation using steel tuning forks}

The validity and applicability of the equations for resonance frequency and quality factor discussed in Secs. 4 and 5 is demonstrated by an experimental investigation using conventional steel tuning forks with circular and rectangular cross sections but with the same nominal resonance frequency $(440 \mathrm{~Hz})$ in air. The usage of tuning forks was motivated by their simple and

- Measuring principle
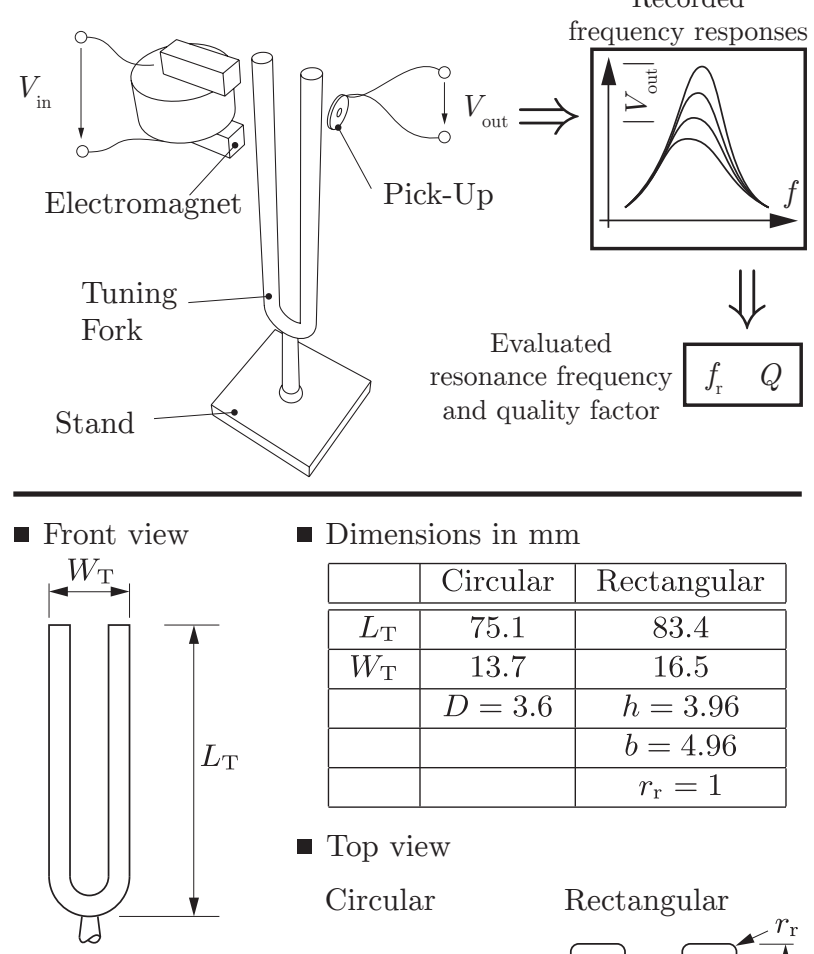

- Dimensions in $\mathrm{mm}$

\begin{tabular}{|c|c|c|}
\hline & Circular & Rectangular \\
\hline \hline$L_{\mathrm{T}}$ & 75.1 & 83.4 \\
\hline$W_{\mathrm{T}}$ & 13.7 & 16.5 \\
\hline & $D=3.6$ & $h=3.96$ \\
\hline & & $b=4.96$ \\
\hline & & $r_{\mathrm{r}}=1$ \\
\hline
\end{tabular}

- Top view Circular

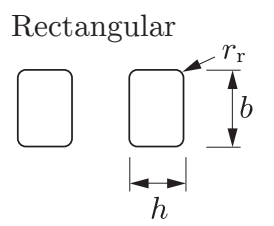

Figure 3: Measuring principle and geometries of the circular and rectangular cross-sectioned tuning fork. The frequency response of a fully immersed, ferromagnetic tuning fork is recorded by electromagnetic excitation and readout. From these frequency responses, resonance frequencies and quality factor are evaluated. 


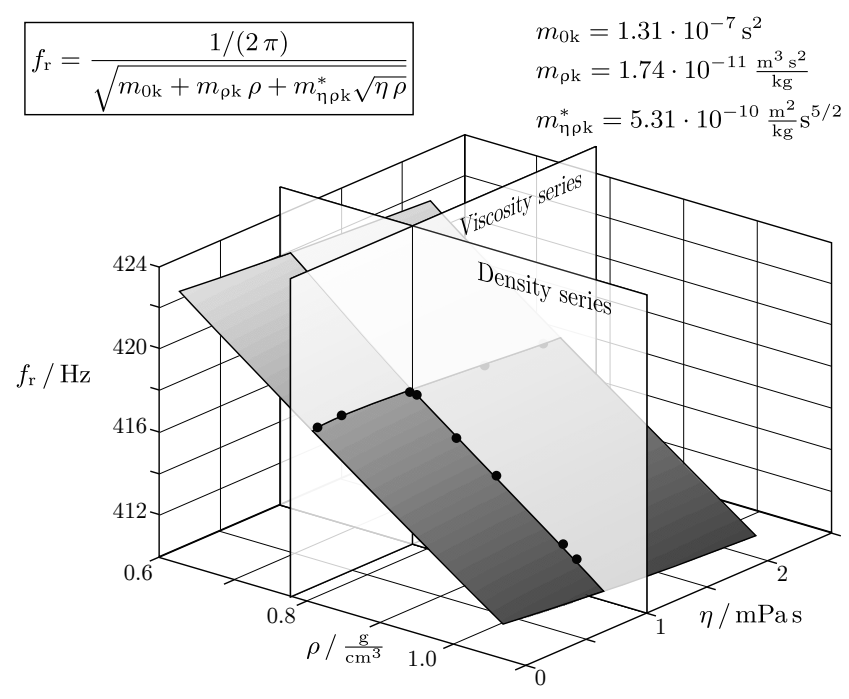

(a) Resonance frequency

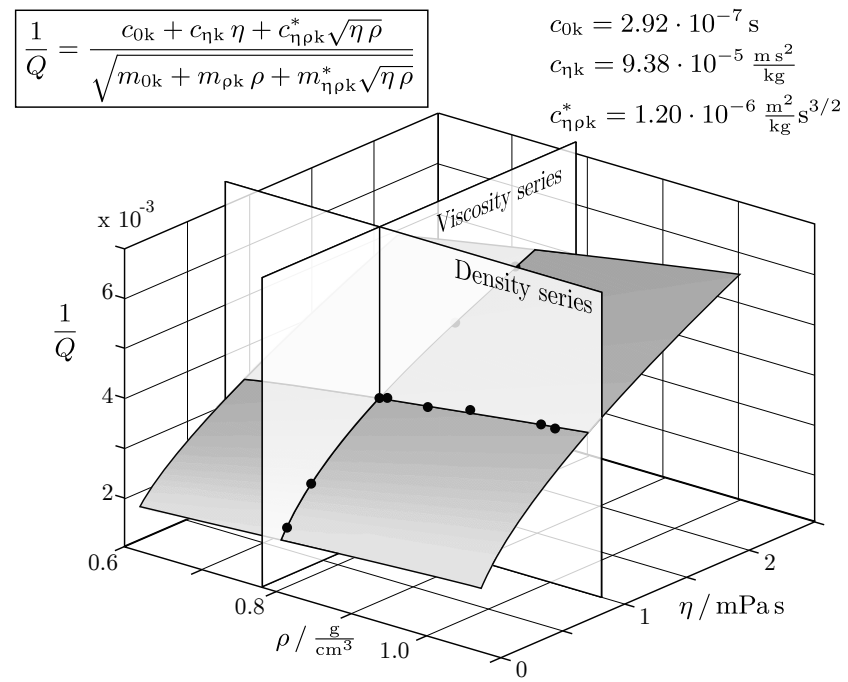

(b) Damping factor

Figure 4: Modeled and measured values for resonance frequency and quality factor. The shaded surfaces for $f_{\mathrm{r}}$ and $Q$ were obtained by evaluating Eqs. 29 and 30 for $f_{\mathrm{r}}$ and $Q$ for identified parameters for the circular tuning fork. The intersection lines on the shaded surfaces with the planes entitled density and viscosity series indicate the values which would be obtained for $f_{\mathrm{r}}$ and $Q$ for constant $\rho$ and $\eta$, respectively. In this case, the viscosity series consist of five aceteone-isopropanol solutions with mass densities of roughly $0.78 \mathrm{mg} / \mathrm{cm}^{3}$ and covering a viscosity range of $0.2 \mathrm{mPa}$ to $2 \mathrm{mPa}$. The density series consist of five solutions with a viscosity of $1 \mathrm{mPa}$ and mass densities of $0.78 \mathrm{mg} / \mathrm{cm}^{3}$ to $1 \mathrm{mg} / \mathrm{cm}^{3}$. The measured points are indicated by the black dots on the shaded surfacs. A more detailed illustration of measured values for $f_{\mathrm{r}}$ and $D$ obtained with the viscosity and mass density series is given in Fig. 5 . Note that here, $1 / Q$ (instead of $Q$ ) is plotted for better visibility of the surface plot.

well defined geometry as well as their stability of resonance frequency and low cross-sensitivity to temperature. Due to these conditions, the results obtained with the tuning forks are expected to yield good accordance with the model. The circular tuning fork was chosen to meet the requirements for the oscillating cylinder, Sec. 3.3 whereas the rectangular tuning fork was selected to demonstrate the applicability of the model for oscillating structures other than plates, cylinders and spheres. The model has furthermore been tested for other sensor types (not only from our own work) described in Sec. 6.2.

The measuring principle as well as the dimensions of the used steel tuning forks are shown in Fig. 3. Each tuning fork was put in a glass tube (not depicted) which was sealed at both sides of the tube after filling it with the sample liquid. For excitation, an electromagnet was placed (outside of the tube) at the end of one of the ferromagnetic tuning fork's prongs. For measuring the oscillation of the tuning fork, an electrodynamic pick-up consisting of a coil carrying a permanent magnet in its center was placed at the other prong's end. By sweeping the excitation current's frequency, the tuning forks' frequency responses containing the tuning forks' fundamental mode were recorded upon immersion in various sample liquids at controlled temperatures of $25 \pm 0.1^{\circ} \mathrm{C}$. From these frequency responses, resonance frequencies and quality factors were evaluated with a fitting procedure presented in [34].

The dependence of the resonance frequency as well as the quality factor on both, the liquid's viscosity and mass density was already shown in the equations for the models discussed in Secs. 4 and 5 (generalized and simplified model). To easier distinguish the tuning forks' responses to mass density and viscosity and to allow plotting $f_{r}(\eta), Q(\eta), f_{r}(\rho)$ and $Q(\rho)$ in a two dimensional plot, two series of liquids have been prepared. The mass density series with a nominal viscosity of $1 \mathrm{mPa}$ and the viscosity series with a nominal mass density of $0.78 \mathrm{mg} / \mathrm{cm}^{3}$. An illustration of $f_{r}(\eta, \rho)$ and $1 / Q(\eta, \rho)$ in the investigated range of viscosities and mass densities is shown Fig. 4.

The response to viscosity was investigated by evaluating both tuning forks' quality factors and resonance frequencies in five acetone-isopropanol solutions covering a viscosity range from $0.2 \mathrm{mPas}$ to $2 \mathrm{mPas}$ for mass densities of roughly $0.78 \mathrm{mg} / \mathrm{cm}^{3}$ at $25^{\circ} \mathrm{C}$. After mixing, the viscosity and mass density of these solutions were measured with an Anton Paar

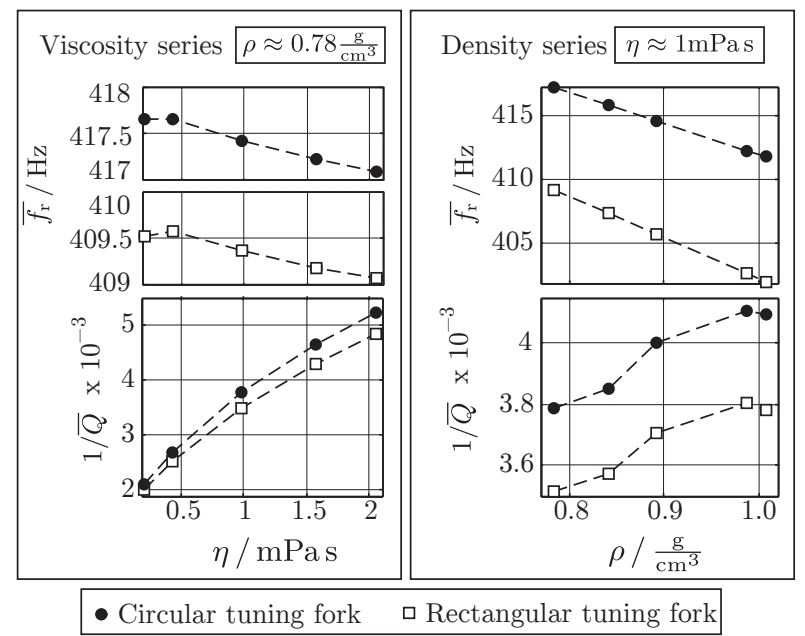

Figure 5: Measured, averaged damping and resonance frequency for a circular and a rectangular tuning fork versus viscosity and mass density. 
SVM 3000 viscometer.

For investigating the response to mass density, five solutions using acetone, isopropanol, ethanol, DI-water and glycerol were prepared. The liquids were mixed to obtain almost constant viscosities of $1 \mathrm{mPas}$ but mass densities between $0.78 \mathrm{mg} / \mathrm{cm}^{3}$ and $1 \mathrm{mg} / \mathrm{cm}^{3}$ at $25^{\circ} \mathrm{C}$. The values for viscosity and mass density of these solutions were again determined with the SVM 3000 viscometer.

The values for the inverse of the quality factor $1 / \bar{Q}$ and the resonance frequency $\bar{f}_{\mathrm{r}}$, averaged over 100 repeated measurements for every liquid from the viscosity and mass density series are depicted in Fig. 5 for the circular and rectangular tuning fork, respectively. This illustration of $f_{\mathrm{r}}(\eta), 1 / Q(\eta), f_{\mathrm{r}}(\rho)$ and $Q(\rho)$ correspond to the measurements in the viscosity series and mass density series planes in Fig. 4.

\subsection{Application to results from literature}

To investigate the applicability of the model not only for tuning forks but furthermore to the work of other groups, the model was tested for other sensors where sufficient data for $f_{\mathrm{r}}$, $Q, \eta$ and $\rho$, was provided in tabulated form. There, the liquids' viscosities and mass densities were not split up in viscosity and mass density series as for the investigation of the tuning forks in this work. In [2] a single-crystal silicon cantilever operated at $5 \mathrm{kHz}$ approximately, with a length of $397 \mu \mathrm{m}$, a width of $29 \mu \mathrm{m}$, and a thickness of $2 \mu \mathrm{m}$ was investigated in gaseous and liquid environments. An in-plane vibrating silicon platelet [16] supported by four silicon beams, featuring an overall size of $1.3 \mathrm{~mm}$ in lenght, $100 \mu \mathrm{m}$ in width and $20 \mu \mathrm{m}$ in height was examined in 9 different liquids at operational frequencies of roughly $10 \mathrm{kHz}$. In [35] a millimeter-sized quartz tuning fork resonating at $30 \mathrm{kHz}$ as well as a $1 \mathrm{~mm}$ long, $250 \mu \mathrm{m}$ wide AlN platelet actuated in an extensional mode at roughly $4 \mathrm{MHz}$ was presented.

From our own work, we further investigated the applicability of the model to a U-shaped wire sensor and a spiral spring sensor. The U-shaped wire sensor [36], consisting of a tungsten wire with a diameter of $400 \mu \mathrm{m}$ which is bent to the shape of a ' $U$ ', features an overall size of $12 \mathrm{~mm}$ in length and $6 \mathrm{~mm}$ in width and is operated at $930 \mathrm{~Hz}$ in liquids. The frequency responses were recorded in eleven DI-water-glycerol mixtures. The spiral spring sensor, see [37], with a length of $35 \mathrm{~mm}$ was tested in 17 liquids at operational frequencies of $630 \mathrm{~Hz}$ in liquids.

\subsection{Comparison of modeled and experimental results}

The relative deviations $\delta f_{\mathrm{r}}$ and $\delta Q$ of the modeled values for $f_{\mathrm{r}}$ and $Q$ with respect to the measured values $f_{\mathrm{r}}^{\mathrm{M}}$ and $Q^{\mathrm{M}}$ are used to show the deviation of the modeled result from the measured value. In case of the circular and rectangular tuning fork, as well as for the U-shaped wire and the spiral spring sensor, which are all from our own work, the mean values for 100 repeated measurements for $f_{\mathrm{r}}^{\mathrm{M}}$ and $Q^{\mathrm{M}}$ have been used. For the other sensors, the reported values have been taken. The relative root mean square (rms) deviations $\delta f_{\mathrm{r} \text {, rms }}$ and $\delta Q_{\mathrm{rms}}$ are used as a figure of merit to judge the applicability of the particular model for resonance frequency and quality factor. The relative deviations and the relative rms deviations were evaluated as follows:

$$
\delta X=\frac{X-X^{\mathrm{M}}}{X^{\mathrm{M}}} \quad \text { and } \quad \delta X_{\mathrm{rms}}=\sqrt{\frac{1}{N} \sum_{i=1}^{N}\left(\frac{X_{i}-X_{i}^{\mathrm{M}}}{X_{i}^{\mathrm{M}}}\right)^{2}}
$$

where $X$ stands either for $f_{\mathrm{r}}$ or $Q$ and $N$ is the number of investigated liquids. The such evaluated results are depicted in Appendix B in Fig. B.6 for eight different sensors.

In total, 77 values for the relative deviations for $f_{\mathrm{r}}$ and $Q$ for both models were evaluated, for the eight investigated sensors. By this investigation, ranges of $\eta=0.21 \ldots 215.7 \mathrm{mPa} \mathrm{s}$, $\rho=0.68 \ldots 1.6 \mathrm{~g} / \mathrm{cm}^{3}, f_{\mathrm{r}} \approx 400 \ldots 3.85 \cdot 10^{6} \mathrm{~Hz}$ and $Q=$ $1.02 \ldots 498.99$ in liquids, have been covered.

The comparison of the relative rms deviations of the models for the resonance frequency Eq. 27 (generalized model) and Eq. 29 (simplified model) shows, that the generalized model in general yields slightly better results than the simplified model. The total rms deviation (for the 77 values) for the resonance frequency is $1.01 \cdot 10^{-3}$ for the generalized model and $1.66 \cdot 10^{-3}$ for the simplified model. In some cases, (circular tuning fork, rectangular tuning fork, U-shaped wire) the simplified model yields negligibly better results. The best fitting results and the highest deviations were obtained in case of the rectangular tuning fork $\left(8.94 \cdot 10^{-6}\right.$ and $\left.8.91 \cdot 10^{-6}\right)$ and the U-shaped wire $\left(2.05 \cdot 10^{-3}\right)$ as well as the silicon cantilever $\left(4.65 \cdot 10^{-3}\right)$, respectively.

Concerning the relative deviations of the values of modeled and measured results for the quality factor, the simplified model Eq. 30 yields in general better results than the generalized model Eq. 28. The total relative rms deviations for the quality factors are $32.44 \cdot 10^{-3}$ for the generalized model and $31.45 \cdot 10^{-3}$ for the simplified model, respectively. The lowest relative deviations are obtained with the silicon platelet $\left(2.50 \cdot 10^{-3}\right.$ and $4.08 \cdot 10^{-3}$ ) whereas the AlN platelet yields the highest relative deviations in case of both models $\left(79.20 \cdot 10^{-3}\right.$ and $\left.78.93 \cdot 10^{-3}\right)$.

\section{Conclusion}

Based on the representation of a mass density and viscosity sensor's characteristic resonant mode by a lumped element oscillator and considering the fluid forces of oscillating plates, spheres and cylinders on the mechanical resonator, generalized reduced order equations for resonance frequency and damping factor were obtained. As the generalized model yielded implicit equations, which might be troublesome in some cases, a simplified model has been formulated, neglecting the frequency dependence of the fluid forces acting on the resonator. For the resonance frequency the difference between both approaches is negligible, whereas in case of the quality factor, the simplified model in general yielded better results. This finding, which needs further investigation, might have different reasons. Measurement errors, resulting e.g., from varying temperatures, not perfectly cleaned sensors, unstable measurement setups and outliers as well as errors resulting from the evaluation of $f_{\mathrm{r}}$ and $Q$ from the frequency response are just a few examples for potential reasons. 
A first investigation of the applicability of the model using the experimental results of conventional steel tuning forks with circular and rectangular cross-sections and six further recently published sensors showed first, that the model can be also applied for resonant sensors not exactly meeting the geometrical requirements (plate, sphere, cylinder) considered in the presented model. Second, the experimental results showed good accordance with the fitted model. The achieved values for the relative rms deviations over 77 investigated measurements are better than $1.7 \cdot 10^{-3}$ for $\delta f_{\mathrm{r}, \mathrm{rms}}$ and $3.3 \cdot 10^{-2}$ for $\delta Q_{r m s}$.

Thus, the presented equations appear suitable for a general description of a resonant mass density and viscosity sensor's performance even if slight deviations of experimental results might be observed. This furthermore allows comparison of sensors of different types, geometries, fabrication technologies as well as actuation and readout principles.

\section{Acknowledgment}

We are indebted to the Austrian COMET program (Austrian Centre of Competence in Mechatronics, ACCM) for the financial support. We also want to thank Bernhard Mayrhofer and Johann Katzenmayer for their help and excellent assistance.

\section{Appendix A. Parameter fit}

Assuming that resonance frequencies $f_{\mathrm{r}, i}$ and quality factors $Q_{i}$ from at least three measurements in liquids with viscosities $\eta_{i}$ and $\rho_{i}$ are available, all parameters in Eqs. $27-30$ can be determined by a linear least squares fit as follows. All four equations can be brought into the form

$$
\mathbf{b}=\mathbf{A} \cdot \mathbf{x}
$$

where $\mathbf{x}$ is a vector with the unknown parameters for which the least squares solution reads [38]:

$$
\mathbf{x}=\left(\mathbf{A}^{\mathrm{T}} \cdot \mathbf{A}\right)^{-1} \cdot \mathbf{A}^{\mathrm{T}} \cdot \mathbf{b}
$$

\section{Appendix A.1. Generalized model}

The vectors of unknowns $\left[m_{0 \mathrm{k}}, m_{\rho \mathrm{k}}, m_{\eta \rho \mathrm{k}}\right]^{\mathrm{T}}$ and $\left[c_{0 \mathrm{k}}, c_{\eta \mathrm{k}}, c_{\eta \rho \mathrm{k}}\right]^{\mathrm{T}}$ of the generalized model can be determined independently with known values for $\omega_{0, i}=2 \pi f_{\mathrm{r}, i}, Q_{i}, \eta_{i}$ and $\rho_{i}$. The equations for $\omega_{0}$ and $Q$ are rearranged to allow formulating matrix equations of the form of Eq. A.1 as follows:

- Equation 27 is rearranged to

$$
\frac{1}{\omega_{0}^{2}}=m_{0 \mathrm{k}}+m_{\rho \mathrm{k}} \rho+m_{\eta \rho \mathrm{k}} \sqrt{\frac{\eta \rho}{\omega_{0}}}
$$

and thus, the least squares solutions for the the parameters in $\mathbf{x}=\left[m_{0 \mathrm{k}}, m_{\rho \mathrm{k}}, m_{\eta \rho \mathrm{k}}\right]^{\mathrm{T}}$ can be found using Eq. A.2 with

$$
\mathbf{b}=\left[\frac{1}{\omega_{0, i}^{2}}\right], \quad \mathbf{A}=\left[1, \rho_{i}, \sqrt{\frac{\eta_{i} \rho_{i}}{\omega_{0, i}}}\right]
$$

- Equation 28 brought into the form

$$
\frac{1}{Q}=\omega_{0} \cdot\left(c_{0 \mathrm{k}}+c_{\eta \mathrm{k}} \eta+c_{\eta \rho \mathrm{k}} \sqrt{\omega_{0} \eta \rho}\right)
$$

shows that the least squares solutions for the parameters of the vector of unknowns $\mathbf{x}=\left[c_{0 \mathrm{k}}, c_{\eta \mathrm{k}}, c_{\eta \rho \mathrm{k}}\right]^{\mathrm{T}}$ can be found using

$$
\mathbf{b}=\left[\frac{1}{Q_{i}}\right], \quad \mathbf{A}=\left[\omega_{0, i}, \omega_{0, i} \eta_{i}, \omega_{0, i} \sqrt{\omega_{0, i} \eta_{i} \rho_{i}}\right]
$$

and Eq. A.2

\section{Appendix A.2. Simplified model}

Similar to the procedure above, the parameters of the simplified model can be determined by a linear least squares fit, whereas in this case, for calculating $\left[c_{0 \mathrm{k}}, c_{\eta \mathrm{k}}, c_{\eta \rho \mathrm{k}}^{*}\right]^{\mathrm{T}}$, the parameters for the resonance frequency $\left[m_{0 \mathrm{k}}, m_{\rho \mathrm{k}}, m_{\eta \rho \mathrm{k}}^{*}\right]^{\mathrm{T}}$ have to be known, i.e., determined first.

- The parameters of Eq. 29 are fitted using Eq. A.2, where $\mathbf{x}=\left[m_{0 \mathrm{k}}, m_{\rho \mathrm{k}}, m_{\eta \rho \mathrm{k}}^{*}\right]^{\mathrm{T}}$ and

$$
\mathbf{b}=\left[\frac{1}{\omega_{0, i}^{2}}\right], \quad \mathbf{A}=\left[1, \rho_{i}, \sqrt{\eta_{i} \rho_{i}}\right] .
$$

- To determine the least squares values of $\mathbf{x}=\left[c_{0 \mathrm{k}}, c_{\eta \mathrm{k}}, c_{\eta \rho \mathrm{k}}^{*}\right]^{\mathrm{T}}$

$$
\mathbf{b}=\left[\frac{1}{Q_{i}}\right], \quad \mathbf{A}=\left[\omega_{0 \mathrm{f}, i}, \omega_{0 \mathrm{f}, i} \eta_{i}, \omega_{0 \mathrm{f}, i} \sqrt{\eta_{i} \rho_{i}}\right]
$$

with

$$
\omega_{0 \mathrm{f}, i}=\frac{1}{\sqrt{m_{0 \mathrm{k}}+m_{\rho \mathrm{k}} \rho_{i}+m_{\eta \rho \mathrm{k}}^{*} \sqrt{\eta_{i} \rho_{i}}}} .
$$

are used and substituted in Eq. A.2.

\section{Appendix B. Model evaluation using experimental results}

Figure B.6 shows the relative deviations $\delta f_{\mathrm{r}}$ and $\delta Q$ of modeled from measured values for resonance frequency and quality factor for eight different sensors versus $\eta$ and $\rho$. The references are given in each plot. The marker $\bullet$ indicates the values for the generalized model whereas the marker $\circ$ designates the values for the simplified model. Furthermore, the relative rms deviations $\delta f_{\mathrm{r} \text {,rms }}$ and $\delta Q_{\mathrm{rms}}$ are given for each sensor for the generalized and the simplified model. 

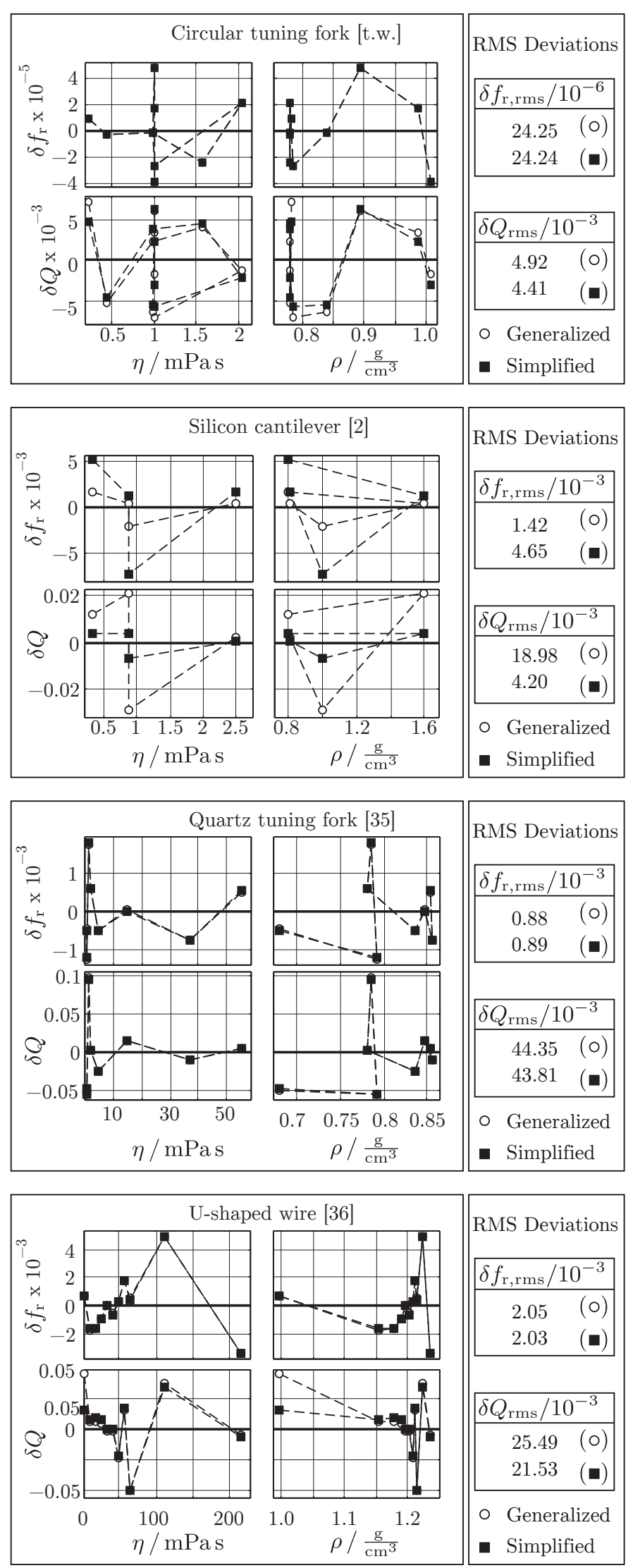

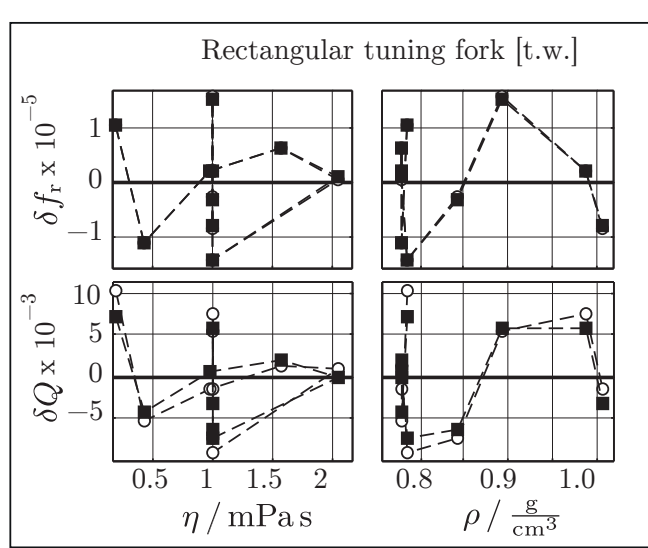

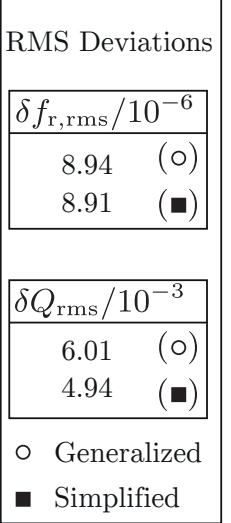
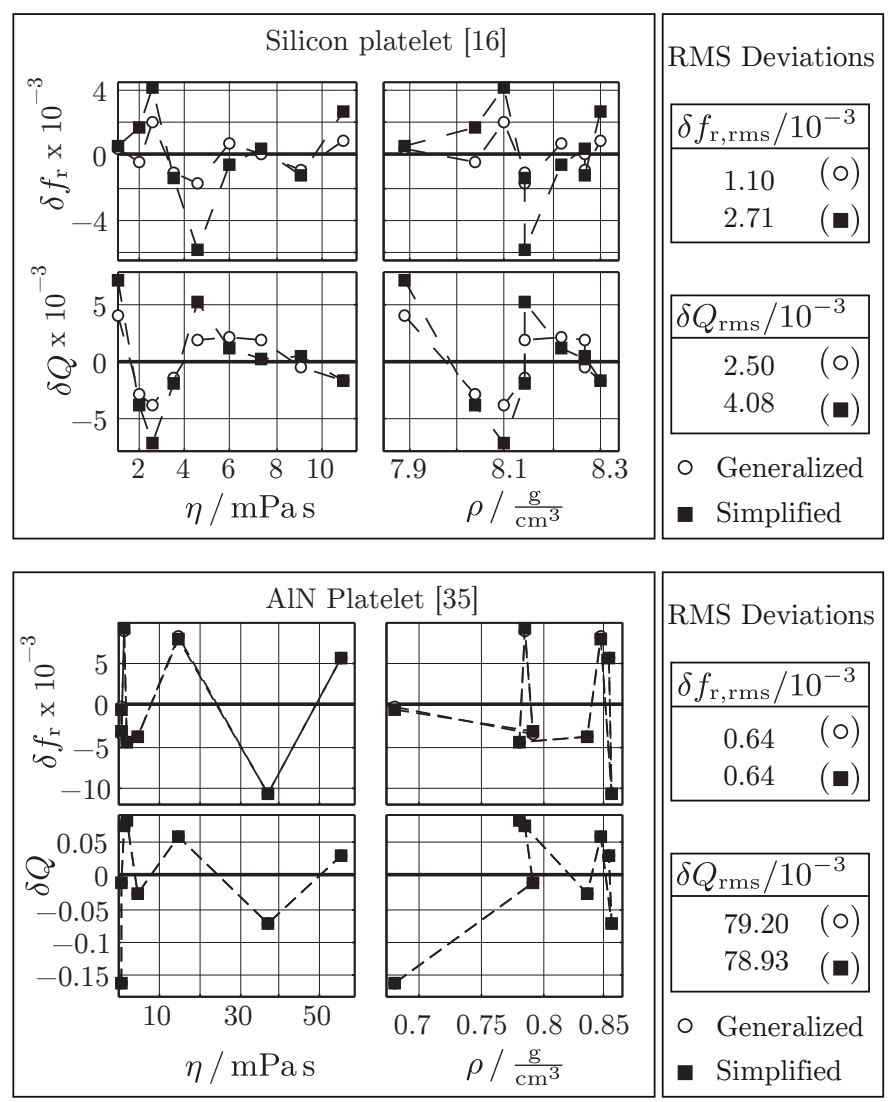

Figure B.6: Relative deviations of modeled from measured results. a designates the generalized model whereas $\bigcirc$ represents the simplified model. In some cases, only one of both markers is visible, as both models yield similar results. 


\section{References}

[1] B. Jakoby, R. Beigelbeck, F. Keplinger, F. Lucklum, A. Niedermayer, E. K. Reichel, C. Riesch, T. Voglhuber-Brunnmaier, B. Weiss, Miniaturized sensors for the viscosity and density of liquids - performance and issues, IEEE transactions on ultrasonics, ferroelectrics, and frequency control 57 (1) (2010) 111-120. doi:10.1109/TUFFC.2010.1386.

[2] S. Boskovic, J. W. M. Chon, P. Mulvaney, J. E. Sader, Rheological measurements using microcantilevers, Journal of Rheology 46 (4) (2002) 891-899.

[3] S. J. Martin, V. E. Granstaff, G. C. Frye, Characterization of a quartz crystal microbalance with simultaneous mass and liquid loading, Anal. Chem. 63 (1991) 2272-2281.

[4] R. Thalhammer, S. Braun, B. Devcic-Kuhar, M. Gröschl, F. Trampler, E. Benes, H. Nowotny, M. Kostal, Viscosity sensor utilizing a piezoelectric thickness shear sandwich resonator, IEEE Trans. Ultrasonics Ferroel. Frequ. Contr. 45 (5) (1998) 1331-1340.

[5] L. Matsiev, J. Bennett, O. Kolosov, High precision tuning fork sensor for liquid property measurements, IEEE Ultrasonics Symposium (2005) 1492-1495.

[6] I. Dufour, A. Maali, Y. Amarouchene, et al., The microcantilever: A versatile tool for measuring the rheological properties of complex fluids, Journal of Sensors 2012. doi:10.1155/2012/719898.

[7] C. Riesch, A. Jachimowicz, F. Keplinger, E. K. Reichel, B. Jakoby, A novel sensor system for liquid properties based on a micromachined beam and a low-cost optical readout, Proceedings IEEE Sensors (2007) 872875.

[8] E. K. Reichel, C. Riesch, B. Weiss, B. Jakoby, A vibrating membrane rheometer utilizing electromagnetic excitation, Sensors and Actuators A: Physical 145 (2008) 349-353.

[9] C. Castille, I. Dufour, C. Lucat, Longitudinal vibration mode of piezoelectric thick-film cantilever-based sensors in liquid media, Applied Physics Letters 96 (15) (2010) 154102. doi:10.1063/1.3387753.

[10] F. J. P. Caetano, J. M. N. A. Fareleira, C. M. B. P. Oliveira, W. A. Wakeham, Validation of a vibrating-wire viscometer: Measurements in the range of 0.5 to 135 mpa s, Journal of Chemical \& Engineering Data 50 (1) (2005) 201-205.

[11] M. Heinisch, E. K. Reichel, I. Dufour, B. Jakoby, Tunable resonators in the low khz range for viscosity sensing, Sensors and Actuators A: Physical 186 (2012) 111-117. doi:http://dx.doi.org/10.1016/j.sna.2012.03.009.

[12] P. Rust, D. Cereghetti, J. Dual, A micro-liter viscosity and density sensor for the rheological characterization of dna solutions in the kilo-hertz range, Lab Chip 13 (24) (2013) 4794-4799. doi:10.1039/C3LC50857A.

[13] M. Heinisch, E. K. Reichel, B. Jakoby, U-shaped wire based resonators for viscosity and mass density sensing, Proc. of. SENSOR 2013 OPTO 2013 IRS2 2013.

[14] M. Heinisch, E. K. Reichel, I. Dufour, B. Jakoby, A resonating rheometer using two polymer membranes for measuring liquid viscosity and mass density, Sensors and Actuators A: Physical 172 (1) (2011) 82-87. doi:10.1016/j.sna.2011.02.031.

[15] P. Peiker, E. Oesterschulze, Impact of the miniaturization on the response of a hybrid diaphragm resonator considering its finite support, Proc. of the Nanomechanical Sensing Workshop (2013) pp. 127-128.

[16] C. Riesch, E. K. Reichel, A. Jachimowicz, J. Schalko, P. Hudek, B. Jakoby, F. Keplinger, A suspended plate viscosity sensor featuring inplane vibration and piezoresistive readout, J. Micromech. Microeng. 19 (2009) 075010. doi:10.1088/0960-1317/19/7/075010.

[17] E. K. Reichel, C. Riesch, F. Keplinger, C. E. A. Kirschhock, B. Jakoby, Analysis and experimental verification of a metallic suspended plate resonator for viscosity sensing, Sensors and Actuators A: Physical 162 (2010) 418-424. doi:10.1016/j.sna.2010.02.017.

[18] A. Abdallah, M. Heinisch, B. Jakoby, Measurement error estimation and quality factor improvement of an electrodynamic-acoustic resonator sensor for viscosity measurement, Sensors and Actuators A: Physical 199 (2013) 318-324.

[19] G. Wingqvist, J. Bjurstrom, L. Liljeholm, I. Katardjiev, A. L. Spetz, Shear mode aln thin film electroacoustic resonator for biosensor applications, Proceedings IEEE Sensors (2005) 492-495.

[20] X. Huang, S. Li, J. Schultz, Q. Wang, Q. Lin, A capacitive mems viscometric sensor for affinity detection of glucose, Journal of Microelectromechanical Systems 18 (6) (2009) 1246-1254.
[21] C. Vančura, J. Lichtenberg, A. Hierlemann, F. Josse, Characterization of magnetically actuated resonant cantilevers in viscous fluids, Applied Physics Letters 87 (16) (2005) 162510.

[22] J. H. Seo, O. Brand, High q-factor in-plane-mode resonant microsensor platform for gaseous/liquid environment, Journal of Microelectromechanical Systems 17 (2) (2008) 483-493.

[23] L. A. Beardslee, A. M. Addous, S. Heinrich, F. Josse, I. Dufour, O. Brand, Thermal excitation and piezoresistive detection of cantilever in-plane resonance modes for sensing applications, Journal of Microelectromechanical Systems 19 (4) (2010) 1015-1017.

[24] A. Rahafrooz, S. Pourkamali, Characterization of rotational mode disk resonator quality factors in liquid, Frequency Control and the European Frequency and Time Forum (FCS), 2011 Joint Conference of the IEEE International (2011) 5 pages.

[25] C. Riesch, E. K. Reichel, F. Keplinger, B. Jakoby, Characterizing vibrating cantilevers for liquid viscosity and density sensing, Journal of Sensors 2008 (Article ID 697062) (2008) 9 pages.

[26] B. N. Johnson, R. Mutharasan, Biosensing using dynamic-mode cantilever sensors: a review, Biosensors and Bioelectronics 32 (1) (2012) $1-18$.

[27] B. A. Bircher, L. Duempelmann, K. Renggli, H. P. Lang, C. Gerber, N. Bruns, T. Braun, Real-time viscosity and mass density sensors requiring microliter sample volume based on nanomechanical resonators, Analytical chemistry 85 (18) (2013) 8676-8683.

[28] W. W. Weaver, S. P. Timoshenko, D. H. Young, Vibration Problems in Engineering, 5th Edition, Wiley, 1990.

[29] M. Heinisch, E. K. Reichel, B. Jakoby, A suspended plate in-plane resonator for rheological measurements at tunable frequencies, in: Proc. Sensor + Test, 2011, pp. 61-66.

[30] L. D. Landau, E. M. Lifshitz, Fluid Mechanics, Butterworth-Heinemann, 1987.

[31] L. Rosenhead, Laminar Boundary Layers: Fluid Motion Memoirs, Clarendon, Oxford, 1963.

[32] E. O. Tuck, Calculation of unsteady flows due to small motions of cylinders in a viscous fluid, Journal of Engineering Mathematics 3 (1) (1969) 29-44.

[33] J. E. Sader, Frequency response of cantilever beams immersed in viscous fluids with applications to the atomic force microscope, Journal of Applied Physics 84 (1998) 64-76.

[34] A. O. Niedermayer, T. Voglhuber-Brunnmaier, J. Sell, B. Jakoby, Methods for the robust measurement of the resonant frequency and quality factor of significantly damped resonating devices, Measurement Science and Technology 23 (8) (2012) 085107.

[35] J. Toledo, T. Manzaneque, J. Hernando-García, J. Vázquez, A. Ababneh, H. Seidel, M. Lapuerta, J. Sánchez-Rojas, Application of quartz tuning forks and extensional microresonators for viscosity and density measurements in oil/fuel mixtures, Microsystem Technologies (2014) 1-9.

[36] M. Heinisch, E. K. Reichel, I. Dufour, B. Jakoby, A u-shaped wire for viscosity and mass density sensing, Sens. Actuators A: Phys. 214 (2014) 245-251.

[37] M. Heinisch, S. Clara, I. Dufour, B. Jakoby, A spiral spring resonator for mass density and viscosity measurements, Proc. Eurosensors XXVIII (2014).

[38] E. K. Chong, S. H. Zak, An introduction to optimization, 2nd Edition, John Wiley \& Sons, 2001.

\section{Biographies}

Martin Heinisch obtained his Dipl.-Ing. (M.Sc.) in Mechatronics from Johannes Kepler University Linz, Austria, in 2009. After his Master studies he went to the University of California, Los Angeles (U.C.L.A.) as a Marshall Plan Scholarship grantee, where he did research in the field of microfluidic applications and self assembling systems. In 2010 he started a Ph.D. program at the Institute for Microelectronics and Microsensors of the Johannes Kepler University Linz, Austria where he is currently working on resonating liquid sensors. 
Thomas Voglhuber-Brunnmaier received the Dipl.-Ing. (M.Sc.) degree in Mechatronics in 2007 and the Dr.techn. (Ph.D.) in May 2013 at the Institute for Microelectronics and Microsensors (IME) at the Johannes Kepler University (JKU) in Linz, Austria. From May 2013 he holds a PostDoc position at the Center for Integrated Sensor Systems (CISS) at the Danube University Krems (DUK), where he works in close cooperation with IME on fluid sensors. His fields of interest are the modeling of micro-sensors, statistical signal processing, numerical methods and analog electronics.

Erwin K. Reichel was born in Linz, Austria, in 1979. He received the Dipl.-Ing. (M.Sc.) degree in mechatronics from Johannes Kepler University, Linz, Austria, in 2006. From 2006 to 2009 he was working on the Ph.D. thesis at the Institute for Microelectronics and Microsensors of the Johannes Kepler University, Linz and graduated in October 2009. Afterwards he held a post-doctoral position at the Centre for Surface Chemistry and Catalysis as well as at the Department for Chemical Engineering, KU Leuven, Belgium until June 2012. Since then he holds a position as university assistant at the Johannes Kepler University Linz. The main research fields are the modeling, design, and implementation of sensors for liquid properties, and monitoring of phase transition in complex solutions.

Isabelle Dufour graduated from Ecole Normale Supérieure de Cachan in 1990 and received the Ph.D. and H.D.R. degrees in engineering science from the University of Paris-Sud, Orsay, France, in 1993 and 2000, respectively. She was a CNRS research fellow from 1994 to 2007, first in Cachan working on the modelling of electrostatic actuators (micromotors, micropumps) and then after 2000 in Bordeaux working on microcantileverbased chemical sensors. She is currently Professor of electrical engineering at the University of Bordeaux and her research interests are in the areas of microcantilever-based sensors for chemical detection, rheological measurements and materials characterisation.

Bernhard Jakoby obtained his Dipl.-Ing. (M.Sc.) in Communication Engineering and his doctoral (Ph.D.) degree in electrical engineering from the Vienna University of Technology (VUT), Austria, in 1991 and 1994, respectively. In 2001 he obtained a venia legendi for Theoretical Electrical Engineering from the VUT. From 1991 to 1994 he worked as a Research Assistant at the Institute of General Electrical Engineering and Electronics of the VUT. Subsequently he stayed as an Erwin Schrödinger Fellow at the University of Ghent, Belgium, performing research on the electrodynamics of complex media. From 1996 to 1999 he held the position of a Research Associate and later Assistant Professor at the Delft University of Technology, The Netherlands, working in the field of microacoustic sensors. From 1999 to 2001 he was with the Automotive Electronics Division of the Robert Bosch GmbH, Germany, where he conducted development projects in the field of automotive liquid sensors. In 2001 he joined the newly formed Industrial Sensor Systems group of the VUT as an Associate Professor. In 2005 he was appointed Full Professor of Microelectronics at the Johannes Kepler University Linz, Austria. He is currently working in the field of liquid sensors and monitoring systems.

\section{List of Figures}

1 Mechanical, lumped elements oscillator, immersed into a liquid. $m_{0}$ : lumped mass, $c_{0}$ : damping coefficient, $k_{0}$ : spring constant, $u(t)$ : displacement, $F_{\text {ex }}(t)$ : excitation force, $F_{\mathrm{F}}(t)$ : fluid force, $f_{\mathrm{r}}$ : resonance frequency, $Q$ : Quality factor, $\eta$ : fluid's dynamic viscosity, $\rho$ : fluid's mass

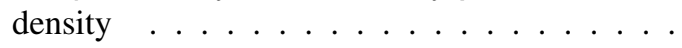

2 Relative deviations of the real and imaginary parts of the approximated hydrodynamic function Eq. 23 from the exact solution Eq. 21. . . .

3 Measuring principle and geometries of the circular and rectangular cross-sectioned tuning fork. The frequency response of a fully immersed, ferromagnetic tuning fork is recorded by electromagnetic excitation and readout. From these frequency responses, resonance frequencies and quality factor are evaluated. . . . . . . . . . .

4 Modeled and measured values for resonance frequency and quality factor. The shaded surfaces for $f_{\mathrm{r}}$ and $Q$ were obtained by evaluating Eqs. 29 and 30 for $f_{\mathrm{r}}$ and $Q$ for identified parameters for the circular tuning fork. The intersection lines on the shaded surfaces with the planes entitled density and viscosity series indicate the values which would be obtained for $f_{\mathrm{r}}$ and $Q$ for constant $\rho$ and $\eta$, respectively. In this case, the viscosity series consist of five aceteone-isopropanol solutions with mass densities of roughly $0.78 \mathrm{mg} / \mathrm{cm}^{3}$ and covering a viscosity range of $0.2 \mathrm{mPa}$ s to $2 \mathrm{mPa}$. The density series consist of five solutions with a viscosity of $1 \mathrm{mPa}$ s and mass densities of $0.78 \mathrm{mg} / \mathrm{cm}^{3}$ to $1 \mathrm{mg} / \mathrm{cm}^{3}$. The measured points are indicated by the black dots on the shaded surfacs. A more detailed illustration of measured values for $f_{\mathrm{r}}$ and $D$ obtained with the viscosity and mass density series is given in Fig. 5. Note that here, $1 / Q$ (instead of $Q$ ) is plotted for better visibility of the surface plot. .

5 Measured, averaged damping and resonance frequency for a circular and a rectangular tuning fork versus viscosity and mass density. . . . . .

B.6 Relative deviations of modeled from measured results. - designates the generalized model whereas $\bigcirc$ represents the simplified model. In some cases, only one of both markers is visible, as both models yield similar results. 